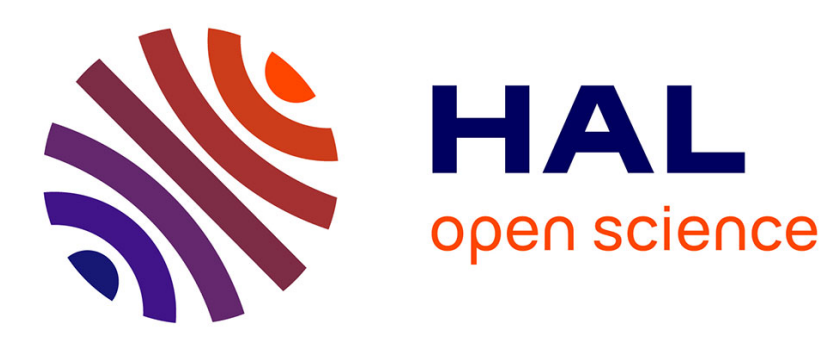

\title{
Relations entre rythme d'apparition des feuilles, nombre total de feuilles et précocité de floraison chez le maïs
}

\author{
Patrick Vincourt
}

\section{To cite this version:}

Patrick Vincourt. Relations entre rythme d'apparition des feuilles, nombre total de feuilles et précocité de floraison chez le maïs. Agronomie, 1984, 4 (8), pp.795-800. hal-00884698

\section{HAL Id: hal-00884698 \\ https://hal.science/hal-00884698}

Submitted on 1 Jan 1984

HAL is a multi-disciplinary open access archive for the deposit and dissemination of scientific research documents, whether they are published or not. The documents may come from teaching and research institutions in France or abroad, or from public or private research centers.
L'archive ouverte pluridisciplinaire HAL, est destinée au dépôt et à la diffusion de documents scientifiques de niveau recherche, publiés ou non, émanant des établissements d'enseignement et de recherche français ou étrangers, des laboratoires publics ou privés. 


\title{
Relations entre rythme d'apparition des feuilles, nombre total de feuilles et précocité de floraison chez le maïs
}

\author{
Patrick VINCOURT ( ${ }^{\mathrm{l}}$ ) \\ I.N.R.A., Station d'Amélioration des Plantes fourragères, F 86600 Lusignan
}

RÉSUMÉ

\begin{abstract}
Les rythmes comparés d'apparition des limbes et des gaines foliaires de maïs sont analysés dans leur relation avec la précocité de floraison et le nombre total de feuilles. Le phyllotherme, somme de température qui sépare l'apparition de deux gaines successives, varie selon le génotype en fonction de l'âge de la plante, en relation à la fois avec la taille du système foliaire et avec sa rapidité de mise en place. La valeur moyenne du phyllotherme au stade jeune pourrait constituer un critère de sélection intéressant pour accroître la vigueur de la plante adulte à précocité constante.
\end{abstract}

Mots clés additionnels : Zea mays, phyllotherme.

The rate of sheath and blade emergence was analyzed in maize, in relation to earliness of flowering and total leaf number. The phyllotherm, defined as the sum of degree days between the emergence of two successive sheaths, was found to depend on the stage of development.

Some correlations were found between the phyllotherm, the total number of leaves and earliness. The mean value of the phyllotherm at the juvenile stage could be used as a selection criterion to improve the vigour of the adult plant for a given earliness of flowering.

Additional key words : Zea mays, phyllotherm.

\section{INTRODUCTION}

Le rythme d'apparition des feuilles est la résultante dynamique des phénomènes de croissance et de développement. Il est impliqué dans des caractéristiques agronomiques importantes telles que la vigueur au stade jeune et la précocité. Les relations entre nombre total de feuilles à la floraison et précocité ont déjà été étudiées par de nombreux auteurs (Allen et al., 1973; Chase \& NANDA, 1977 ; Cross \& Zuber, 1973). D'autre part, lorsque l'on connaît le nombre total de feuilles d'un génotype, il est possible de prévoir son stade de développement à partir du nombre de feuilles déjà apparues (AITKEN, 1976; GAY \& MENETRIER, 1978). Le taux d'apparition des feuilles a été utilisé comme mesure biologique des besoins en température pour prévoir les dates de floraison (TOLLENAAR et al., 1979). HEBERT (1981), à partir d'un diallèle entre lignées cornées et dentées, a mis en évidence l'existence d'aptitudes générales et spécifiques à la combinaison

(1) Adresse actuelle : INRA, Domaine de Melgueil, 34130 Mauguio. pour différents critères de vitesse d'apparition des feuilles. L'effet d'hétérosis avait assez peu d'ampleur sur ces caractères.

De façon variable selon l'année et la synthétique améliorée, la notation visuelle de vigueur, pratiquée au stade « 7-8 feuilles visibles », s'est révélée être un critère de sélection intéressant pour l'amélioration simultanée du rendement et de la teneur en matière sèche à la récolte du maîs ensilage (GALLAIS et al., 1983). C'est dans le but d'appréhender le contenu et les implications de cette notation qu'on analyse ici les relations entre rythme d'apparition des feuilles d'une part, précocité de floraison et nombre total de feuilles d'autre part.

\section{MÉTHODES ET TECHNIQUES}

\section{A. Critères soumis à l'analyse}

Jusqu'à l'initiation de la panicule, l'apex différencie les bourgeons qui donneront les feuilles selon un rythme, le plastochrone, difficilement accessible à l'observation. On peut par contre sans difficultés noter le stade "feuille visible ", où le limbe en croissance apparaît nettement dans le cornet foliaire, et le stade 
« feuille adulte », où la ligule, extrêmité de la gaine, est visible.

Notre objectif a été d'étudier dans quelle mesure les relations entre les chronologies de ces 2 types d'événements pouvaient fournir une indication sur le nombre total de feuilles et la précocité de floraison. Deux types de critères furent donc analysés :

- les variables $D_{n-m}$, sommes de température (en base $6{ }^{\circ} \mathrm{C}$ pour la température du sol à $-10 \mathrm{~cm}$ ) qui séparent les apparitions des gaines $\mathrm{n}$ et $\mathrm{m}$, caractérisent une échelle de temps, celle qui décrit l'âge de la plante par son nombre de feuilles adultes;

- les variables $S_{n-m}$, variations du nombre de feuilles visibles non adultes lorsque la plante passe du stade « $\mathrm{n}$ feuilles adultes » au stade « $\mathrm{m}$ feuilles adultes», décrivent l'apparition des limbes en croissance selon l'échelle de temps définie auparavant.

Nous appellerons phyllotherme d'ordre $n$ la variable $D_{n, n+1}$.

\section{B. Structures génétiques impliquées dans l'expérience}

\section{Essai de 1979}

Neuf variétés (tabl. 1) couvrant une large gamme de précocité, de « $F_{7} \times F_{2}$ » « «INRA 508 », ont été étudiées du semis à la récolte (FOK AH CHUEN, 1979). A partir d'un semis du 10 mai, la notation des stades foliaires a été effectuée sur 20 plantes par parcelle, dans un dispositif à 3 répétitions, à une fréquence assez faible (1 à 2 par semaine) si bien que certains stades n'ont pu être observés sur un nombre suffisant de plantes.

TABLEAU 1

Caractéristiques des génorypes implantés en 1979.

Characteristics of the genotypes tested in 1979.

\begin{tabular}{lccccc}
\hline Génotype & Structure & $\begin{array}{c}\text { Nombre } \\
\text { total de } \\
\text { feuilles }\end{array}$ & $\begin{array}{c}\mathrm{S}_{4-6} \\
(1)\end{array}$ & $\begin{array}{c}\mathrm{D}_{4-6} \\
(1)\end{array}$ & $\begin{array}{c}\text { Floraison } \\
\text { mâle } \\
(2)\end{array}$ \\
\hline $\mathrm{F}_{7} \times \mathrm{F}_{2}$ & $\mathrm{HS}$ & 13,6 & 1,14 & 138 & 756 \\
WH $\times$ WJ & HS & 16,1 & 0,71 & 110 & 800 \\
$\mathrm{BIP}$ & HS & 15,7 & 0,76 & 107 & 775 \\
LG 11 & HTV & 15,1 & 1,04 & 123 & 815 \\
HS 222 & HS & 16,9 & 0,90 & 119 & 868 \\
MONSO & HS & 14,8 & 0,95 & 124 & 853 \\
HTV 216 & HTV & 15,9 & 0,91 & 116 & 845 \\
Prolifique & HD & 20,1 & 0,50 & 102 & 946 \\
INRA 508 & HS & 18,4 & 0,55 & 109 & 883 \\
\hline Ecart-type d'une diffé- & 0,2 & 0,20 & 7 & 8 \\
rence de 2 moyennes & & & & \\
\hline \hline
\end{tabular}

(1) $\mathrm{S}_{4-6}$ (resp. $\mathrm{D}_{4-6}$ ) $6 \mathrm{st}$ la variation du nombre de feuilles visibles non adultes (resp la somme de température en base $6{ }^{\circ} \mathrm{C}$ ) lorsque la plante passe du stade " 4 feuilles adultes » au stade " 6 feuilles adultes ».

$S_{4-6}\left(\right.$ resp. $D_{4-6}$ ) is the variation of the number of just visible leaves (resp. the sum of degree days with the threshold $6^{\circ} \mathrm{C}$ ) between the stages " 4 fully expanded leaves » and « 6 fully expanded leaves".

(2) Exprimée en degrés jours, en base $6^{\circ} \mathrm{C}$, pour la phase levée, floraison.

Expressed in degree days, with the threshold $6^{\circ} \mathrm{C}$ between emergence and flowering time.

\section{Essai de 1980}

Dans le cadre d'une étude sur la prolificité (PHILIPPE, 1980), 6 populations synthétiques ont été examinées (tabl. 2). La synthétique "Sélection $\mathrm{FS}_{10}$ " (respectivement "Sélection $\mathrm{FS}_{11}$ ") provient d'un cycle de sélection massale pour la prolificité et la précocité dans la synthétique $\mathrm{FS}_{10}$ (respectivement $\mathrm{FS}_{11}$ ). Ces sélections ont été effectuées au laboratoire du maïs fourrage de la Station de Lusignan. Le semis a été effectué début juin. 15 à 25 plantes par parcelle ont été examinées dans un dispositif à 3 répétitions. Sur ce matériel génétique, la fréquence des notations a été plus élevée (tous les 2 j) et l'analyse a porté tant sur la variation entre synthétiques que sur la variation intra-synthétique.

\section{RÉSULTATS ET DISCUSSION}

\section{A. Apparition des limbes dans le cornet foliaire}

Lorsque l'échelle de temps utilisée est le nombre de feuilles adultes, la variation du nombre de feuilles en croissance dans le cornet est comparable à la trajectoire d'un projectile dont la portée est le nombre total de feuilles du génotype (fig. 1). L'initiation de la panicule

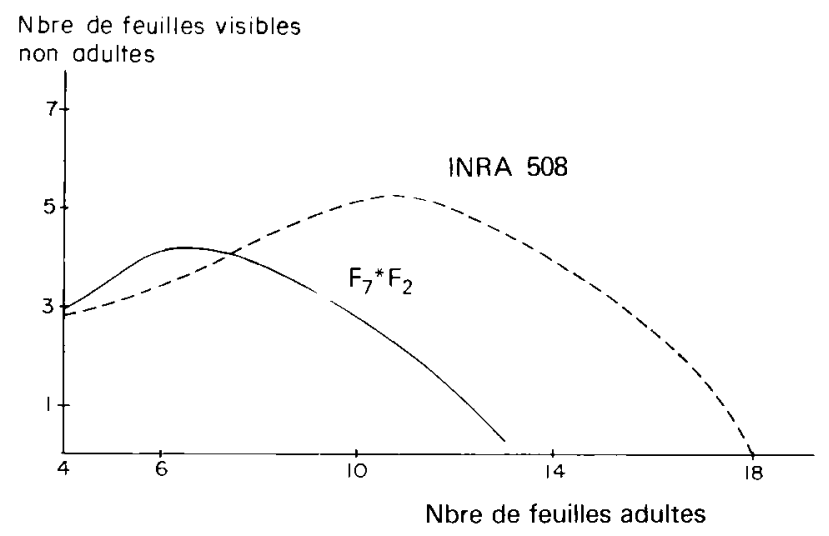

Figure 1

Représentation du rythme d'apparition des feuilles (essai 1979).

Time-free diagram for leaf appearance (1979 trial).

se situe peu après le stade « 3 feuilles adultes » pour « $F_{7} \times F_{2} »$, « 5 feuilles adultes " pour «I.N.R.A. $508 »$. La pente de la courbe au point « 4 feuilles adultes », approximativement mesurée par la variable $\mathrm{S}_{4-6}$, représente un potentiel d'expansion foliaire qui semble maximum une feuille adulte «plus tard» que l'initiation de la panicule. Ce critère pourrait être utilisé comme caractère marqueur du nombre total de feuilles et/ou de la précocité de floraison.

La figure 2 montre en effet qu'il existe une assez bonne liaison (corrélation négative) entre $\mathrm{S}_{4-6}$ et le nombre total de feuilles. Toutefois en 1979, l'estimation de $\mathrm{S}_{4-6}$ est moins précise qu'en 1980 et les différences entre génotypes ne sont significatives qu'au seuil 7 p. 100 (tabl. 1 et 2). 
TABLEAU 2

Caractéristiques des génotypes testés en 1980.

Characteristics of the genotypes tested in 1980.

\begin{tabular}{|c|c|c|c|c|c|}
\hline Génotypes & Structure & $\begin{array}{l}\text { Nombre } \\
\text { total de } \\
\text { feuilles }\end{array}$ & $\begin{array}{l}S_{4-6} \\
(1)\end{array}$ & $\mathrm{D}_{+1-6}$ & $\begin{array}{c}\text { Floraison } \\
\text { mâle } \\
\text { (2) }\end{array}$ \\
\hline FS 10 & $\begin{array}{l}\text { synthétique } \\
\text { prolifique } \\
\text { dentée }\end{array}$ & 20,8 & 0,70 & 138 & 964 \\
\hline Sélection FS 10 & $"$ & 20,2 & 0,78 & 137 & 931 \\
\hline FS 11 & $\begin{array}{l}\text { synthétique } \\
\text { prolifique } \\
\text { cornée }\end{array}$ & 16,4 & 0,81 & 147 & 786 \\
\hline Selection FS 11 & " & 15,7 & 0,81 & 152 & 751 \\
\hline $\operatorname{PrC}$ & $\begin{array}{l}\text { synthétique } \\
\text { prolifique } \\
\text { cornée }\end{array}$ & 13,9 & 1,10 & 163 & 674 \\
\hline Pr D & $\begin{array}{l}\text { synthétique } \\
\text { prolifique } \\
\text { dentée }\end{array}$ & 17,5 & 0,73 & 139 & 819 \\
\hline $\begin{array}{l}\text { Ecart-type d'un } \\
\text { de } 2 \text { moyennes }\end{array}$ & e différence & 0,3 & 0,10 & 4,4 & 9 \\
\hline
\end{tabular}

(1), (2) Voir légende du tableau 1.

(1), (2) As for table 1

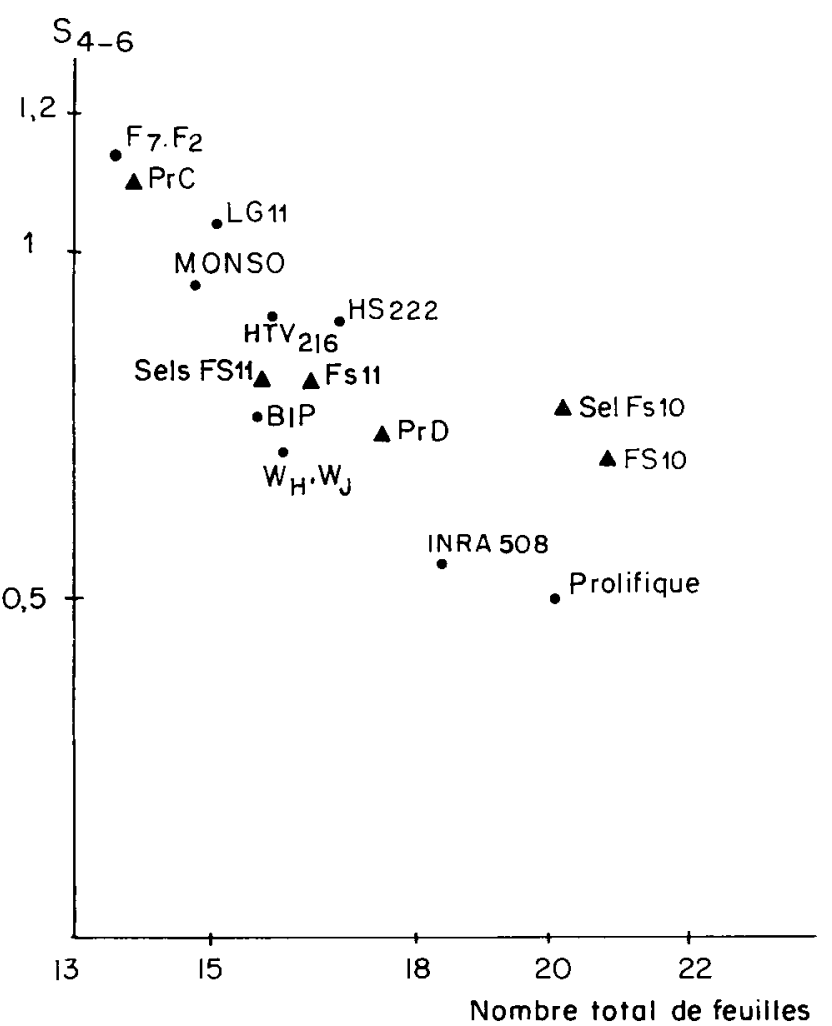

Figure 2

Liaison entre nombre total de feuilles et variation du nombre de feuilles visibles non adultes lorsque le nombre de feuilles adultes passe de 4 à $6\left(S_{4-6}\right)$ (•essai de $1979 ; \mathbf{\Delta}$ essai de 1980).

Relationship between the total leaf number and the $S_{4-6}$ variable (• 1979 trial ; A 1980 trial).

\section{B. Rythme d'apparition des feuilles adultes}

La variable $\mathrm{D}_{4-6}$ a été mesurée en 1979 et en 1980 afin de porter un jugement sur l'échelle de temps utilisée cidessus. Elle peut aisément se substituer à la variable
$\mathrm{S}_{4-6}$, tant en ce qui concerne sa liaison avec le nombre total de feuilles que pour son pouvoir de discrimination des génotypes (tabl. 1 et 2).

En d'autres termes, le nombre de limbes en croissance dans le cornet foliaire varie entre 2 stades foliaires " adultes " comme la somme de température reçue par la plante. Tout se passe comme si l'apparition des limbes était directement reliée à la somme de température, l'apparition des gaines pouvant traduire en outre le stade de développement.

$\mathrm{La}$ liaison entre la somme de température $\mathrm{D}_{4-6}$ et le nombre total de feuilles, observée au niveau des moyennes phénotypiques, est confirmée dans l'analyse intrasynthétique (1980), c'est-à-dire sur des données individuelles (tabl. 3). On peut noter des écarts importants entre les niveaux moyens des variables $D_{4-6}$ évaluées lors des implantations de 1979 et de 1980 . Nous ne pouvons savoir si la différence de conditions générales d'implantation (dates de semis) ou de matériel génétique (matériel prolifique en 1980) en est la cause.

\section{TABLEAU 3}

Régression du nombre total de feuilles sur la variable $D_{4-6}$. (Variation plante a plante intra-synthétique).

Regression of the total number of leaves on the $D_{4-6}$ variable. (Within synthetic variability).

\begin{tabular}{lccccc}
\hline \hline Génotype & $\begin{array}{c}\text { W (1) } \\
\text { avant }\end{array}$ & $\begin{array}{c}\text { W (2) } \\
\text { après }\end{array}$ & $\begin{array}{c}\text { d.d.l. } \\
(3)\end{array}$ & $\begin{array}{c}\text { Coefficient } \\
\text { régression } \\
(4)\end{array}$ & $\begin{array}{c}\text { Signifi- } \\
\text { cation }\end{array}$ \\
\hline FS 10 & 1,44 & 1,26 & 38 & $-3,0$ & $* *$ \\
FS 11 & 2,14 & 2,04 & 40 & $-2,1$ & NS \\
Selection & 1,78 & 1,52 & 41 & $-3,8$ & $* *$ \\
$\begin{array}{l}\text { FS 10 } \\
\text { Selection }\end{array}$ & 1,88 & 1,68 & 39 & $-2,4$ & $*$ \\
$\begin{array}{l}\text { FS 11 } \\
\text { Pr C }\end{array}$ & 1,82 & 1,63 & 64 & $-2,2$ & $* *$ \\
Pr D & 2,03 & 1,83 & 67 & $-2,1$ & $* *$ \\
\hline $\begin{array}{l}\text { Tous géno- } \\
\text { types (5) } \\
\text { confondus }\end{array}$ & 1,87 & 1,65 & 289 & $-2,3$ & $* *$ \\
\hline \hline
\end{tabular}

(1) Carré moyen de la variation du nombre de feuilles. Mean square for total number of leaves.

(2) Carré moyen résiduel après régression. Residual mean square after regression on $\mathrm{D}_{4-6}$.

(3) Nombre de degrés de liberté associé au carré moyen (1). $\mathrm{dF}$ for the mean square (1).

(4) Exprimé en feuilles pour $100^{\circ} \mathrm{C}$ jour (base $6{ }^{\circ} \mathrm{C}$ ). Expressed in leaves for $100^{\circ}$ degree days (threshold $6^{\circ} \mathrm{C}$ ).

(5) Test $\mathrm{F}$ de la différence des pentes $(5$ et 283 d.d.1.) $=0,14$ NS. $F$ test for differences between regression coefficients (with 5 and 283 d.f.) $=0.14 \mathrm{NS}$

\section{Variations du phyllotherme (fig. 3)}

La précision des notations effectuées en 1980 a permis d'estimer les phyllothermes $\mathrm{D}_{4-5}, \mathrm{D}_{5-6}, \mathrm{D}_{6-7}, \mathrm{D}_{7-8}$. En fonction du rang $n$ de la feuille, le phyllotherme d'ordre $n$ suit une courbe en cloche présentant un maximum pour des rangs $\mathrm{n}$ apparemment plus bas chez les génotypes à faible nombre de feuilles que chez les génotypes à nombre de feuilles élevé. Ce maximum pourrait grossièrement correspondre au stade où la panicule achève sa différentiation ( $\mathrm{D}$ dans l'échelle analysée par GAY \& MENETRIER, 1978) et où la tige entre dans sa phase d'élongation active. 


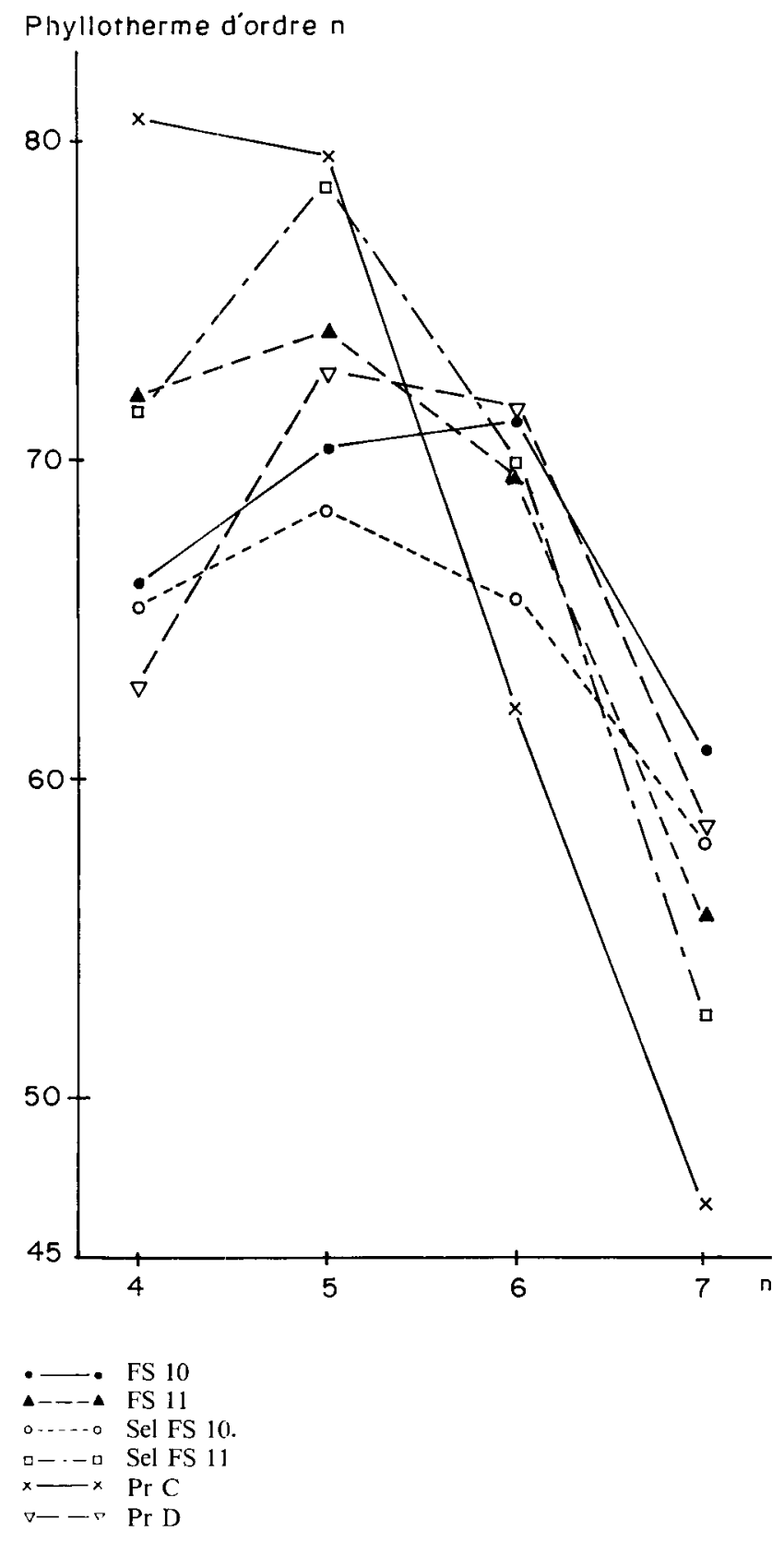

Figure 3

Evolution des sommes de températures $D_{n, n+l}$ en fonction de $n$, rang de la feuille, essai de 1980.

Temperature sums $D_{n, n+1}$ according to $n$, the rank of the leaf.

\section{Nombre total de feuilles et date de floraison : dou- ble signification du phyllotherme}

En 1979 comme en 1980, la liaison entre les sommes de température " levée - floraison mâle » et le nombre total de feuilles est assez étroite $(r=0,880$ pour 9 couples en $1979 ; r=0,999$ pour 6 couples en 1980), au niveau des moyennes phénotypiques. Ce résultat, par ailleurs classique, ne doit pas étonner compte tenu de l'ampleur de la variation génétique observée. Il suggère qu'il n'existe, pour une implantation donnée, que d'assez faibles variations de phyllotherme moyen appa- rent entre génotypes. Il faut cependant noter (fig. 4), d'une part, l'écart moyen entre les 2 implantations, d'autre part, les valeurs assez fortes observées pour ce phyllotherme pour les variétés incluant la lignée $F_{2}$

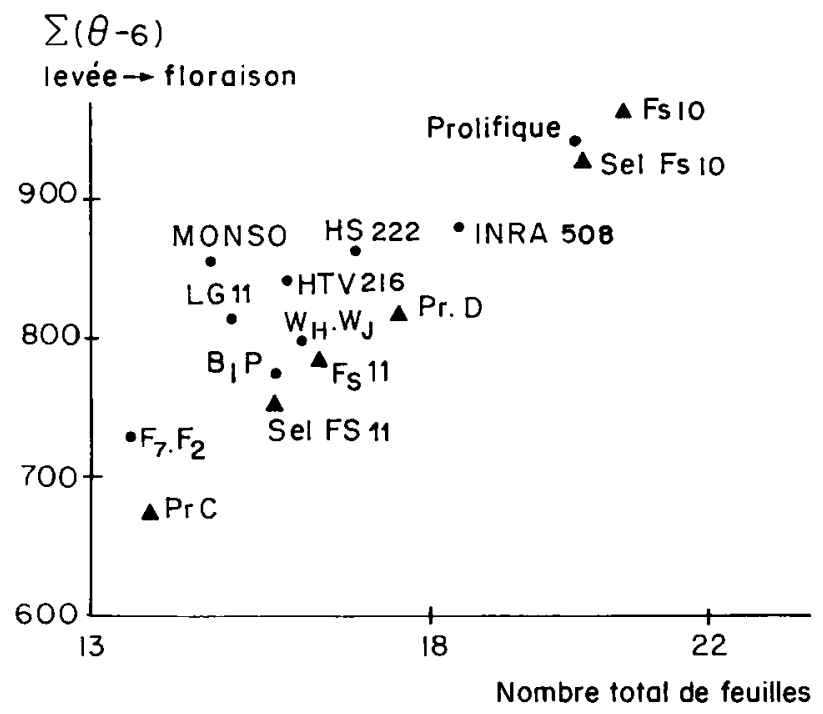

Figure 4

Liaison entre nombre total de feuilles et somme de température levée

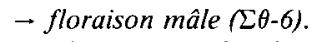

- génotypes implantés en 1979. \ génotypes implantés en 1980.

Relationships between total leaf number and the sum of degree days between emergence and male flowering time.

comme géniteur (« $F_{7} \times F_{2} »$, , HTV $216 »$, « $\left.\mathrm{LG}_{11} »\right)$ et pour la variété « Monso ».

Ce dernier génotype, quoique présentant un nombre de feuilles de génotype précoce, a été sélectionné dans des conditions méridionales et a montré une assez faible vitesse d'implantation à Lusignan en 1979. Les caractéristiques de la lignée $F_{2}$ ont été signalées par ailleurs (DERIEUX, comm. pers.). La valeur plus faible du phyllotherme moyen apparent observé en 1980 pourrait être imputé au caractère de prolificité des génotypes. PhilipPe (1980) a en effet montré qu'à date de floraison égale, les plantes prolifiques présentaient un étage foliaire supplémentaire au niveau des épis. Les différences de dates de semis pourraient également être mises en cause.

D'autre part, l'analyse intra-synthétique montre que la liaison entre la somme de température levée-floraison et le nombre total de feuilles peut être assez faible dans certains contextes génétiques (tabl. 4, synthétiques " $\mathrm{FS}_{11}$ » et «Sel. $\mathrm{FS}_{11}$ "). Particulièrement pour ceuxlà, la connaissance de la variable $\mathrm{D}_{0-4}$, c'est-à-dire de la lenteur de mise en place des toutes premières feuilles, permet d'améliorer l'estimation de la date de floraison, le nombre de feuilles étant connu.

Ainsi ressort la double signification du phyllotherme :

- Au stade jeune et en valeur moyenne apparente sur toute la vie de la plante, il peut constituer un indice de vigueur, de faibles valeurs étant associées à la mise en place rapide du système foliaire ; 


\section{TABLEAU 4}

Apport de la variable $D_{04}$, mesurée au stade jeune, sur la prédiction de la tardiveté.

(Variation plante à plante, intra-synthétique).

Significance of the $D_{0-4}$ variable for the prediction of lateness of flowering time.

(Within synthetic variability).

\begin{tabular}{|c|c|c|c|c|c|c|c|}
\hline Génotype & $\begin{array}{c}\text { d.d.l. } \\
\text { (1) }\end{array}$ & $\begin{array}{l}W_{0} \\
(2)\end{array}$ & $\begin{array}{l}W_{1} \\
(3)\end{array}$ & $\begin{array}{l}W_{2} \\
(4)\end{array}$ & $\begin{array}{c}\mathrm{b} \\
\mathrm{Nb} . \mathrm{F}(5)\end{array}$ & $\mathrm{D}_{0-4}{ }^{\mathrm{b}}(6)$ & $\begin{array}{l}\text { Signifi- } \\
\text { cation (7) }\end{array}$ \\
\hline FS 10 & 38 & 1331 & 869 & 762 & 20,7 & 0,62 & $*$ \\
\hline FS 11 & 40 & 2479 & 2109 & 1523 & 16,3 & 1,22 & $* *$ \\
\hline $\begin{array}{l}\text { Selection } \\
\text { FS } 10\end{array}$ & 41 & 1448 & 1053 & 857 & 20,2 & 0,89 & $* *$ \\
\hline $\begin{array}{l}\text { Selection } \\
\text { FS } 11\end{array}$ & 39 & 1399 & 1386 & 1165 & 6,8 & 0,92 & $* *$ \\
\hline Pr C & 64 & 1604 & 1028 & 735 & 22,6 & 0,79 & $* *$ \\
\hline $\operatorname{Pr} D$ & 67 & 2054 & 754 & 591 & 25,5 & 1,12 & $* *$ \\
\hline
\end{tabular}

(1) Nombre de degrés de liberté affecté à la variation de tardiveté intragénotypique. d.f. for within synthetic variability.

(2) Variation intragénotypique de la tardiveté (avant régression). Mean square for lateness of flowering time (before regression).

(3) Variation résiduelle après prise en compte du nombre de feuilles. Residual mean square after taking in account the number of leaves.

(4) Variation résiduelle après prise en compte du nombre de feuilles et de $D_{0-4}$. Residual mean square after taking in account the number of leaves and $D_{0-4}$.

(5) Coefficient de régression de la variable « $\mathrm{Nb}$. de feuilles». Coefficient of regression for the variable « Number of leaves ».

(6) Coefficient de régression de la variable $\mathrm{D}_{0-4}$. Coefficient of regression for $D_{0-4}$.

(7) Signification du test $\mathbf{F}$ d'apport de la variable $\mathrm{D}_{0-4}$ (après le nombre de feuilles). Significance of $\mathrm{F}$ test for taking in account $\mathrm{D}_{0-4}$ (after the number of leaves).

- Mesuré après la différenciation florale, vers les stades « 4 à 5 feuilles adultes", il est susceptible de donner certaines indications sur la taille de ce système foliaire, de fortes valeurs étant alors associées à des nombres de feuilles peu élevés.

\section{CONCLUSION}

L'examen des rythmes comparés d'émergence des limbes et des gaines jusqu'au stade « 6 feuilles adultes » peut donc fournir des indications à la fois sur l'effectif du système foliaire et la rapidité de sa mise en place, qui sont conjointement des composantes de la précocité de floraison et de la vigueur de la plante. Ces deux caractéristiques sont souvent génétiquement liées de manière négative. Aussi peut-on penser que la notation de vigueur au départ effectuée en sélection intègre de façon empirique, par le jugement sur pied de l'architecture de la plante, l'évolution globale des phyllothermes.

L'accumulation des degrés-jours constitue une hor- loge externe qui semble influencer très directement l'apparition des limbes. Elle sert de moteur au fonctionnement de l'horloge interne qui s'extériorise par l'apparition des gaines. Il est cependant difficile de conclure si le rythme d'apparition des gaines n'est qu'un caractère marqueur de cette horloge ou si, au contraire, le déroulement de la morphogenèse est effectivement temporisé par l'émergence de ces organes.

Des écarts entre phyllothermes moyens apparents ont été observés sans que l'on puisse connaître leur origine avec précision. D'origine génétique, cette variation pourrait être exploitée pour accroître la précocité de floraison de génotypes développant un nombre de feuilles relativement élevé.

Recu le 26 avril 1983. Accepté le 3 mai 1984.

\section{REMERCIEMENTS}

Je tiens à remercier très vivement A. VINCENT (I.N.R.A. Dijon) pour sa contribution à la mise au point de cet article.

\section{RÉFÉRENCES BIBLIOGRAPHIQUES}

Aitken Y., 1976. Non destructive method of estimation of tassel initiation in Maize (Zea mays L.). Aust. Inst. Agric. Sci., 42, 65-66.

Allen J. R., Mc Kee G. W., Mc Gahen J. H., 1973. Leaf number and maturity in hybrid corn. Agron. J., 65, 233-235.
Chase S. S., Nanda D. K., 1967. Number of leaves and maturity classification in Zea mays L. Crop Sci., 7, 431-432.

Cross H. Z., Zuber W. S., 1973. Interrelationship among plant height, number of leaves and flowering dates in Maize. Agron. J., 65, 71-74. 
Easton S., 1976. Eude comparative d'effets génétiques chẽ des plantes diploïdes et tétraploïdes isogéniques de Festuca pratensis Huds. Thèse Doct. d'Etat Sci. Nat., Université Paris Sud, 202 p.

Fok Ah Chuen M., 1979. Variabilité génétique de la levée et de la vigueur au stade jeune chez le mais. Diplôme d'Agronomie approfondie, ENSA Rennes, $55 \mathrm{p}$.

Gallais A., Vincourt P., Bertholleau J. C., 1983. Etude de critères de sélection chez le maîs fourrage : héritabilites, corrélations génetiques et réponse attendue à la sélection. Agronomie, 3 (8), $751-760$.

Gay J. P., 1980. Croissance et développement chez le mais, Publ. Dep. tech. AGPMI, 44 p.
Gay J. P., Menetrier M. A., 1978. Morphogenèse des organes reproducteurs chez, le mais. DOc. AGPM - ITCF, $40 \mathrm{p}$.

Hebert Y., 1981. Analyse quantitative de la vitesse d'apparition des feuilles chez le maïs. Diplôme d'Agronomie approfondie, ENSA Rennes, $70 \mathrm{p}$

Philippe F., 1980. Contribution à l'étude de la prolificité en épis chez le maìs. Diplôme d'Agronomie approfondie, ENSA Montpellier. 83 p.

Tollenaar M., Daynard T. B., Hunter R. B., 1979. Effect of temperature on rate of leaf appearance and flowering date in Maize. Crop Sci., $19,363-366$ 